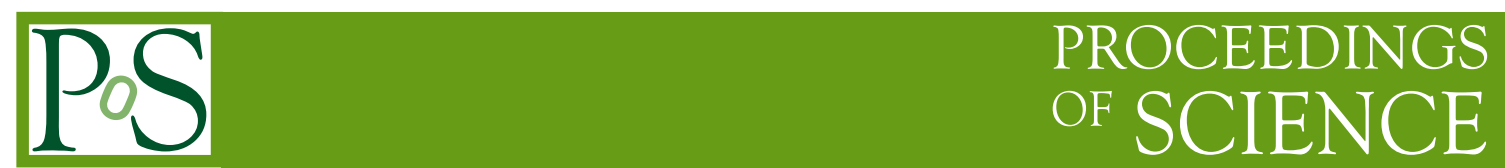

\title{
What Have We Learned From the LHC?
}

Thomas J. LeCompte ${ }^{* \dagger}$

Argonne National Laboratory

E-mail: lecomptedanl.gor

I discuss recent LHC measurements, and how they fit into the larger picture in particle physics.

Theoretical Advanced Study Institute Summer School 2017 "Physics at the Fundamental Frontier"

4 June - 1 July 2017

Boulder, Colorado

\footnotetext{
* Speaker.

$\dagger$ Present address: United States Department of Energy, SC-25 1000 Independence Ave., SW. Washington, DC 20585

(C) Copyright owned by the author(s) under the terms of the Creative Commons 


\section{So What Exactly Have We Learned?}

As a general rule, I try and avoid proceedings. As an experimenter, my talks tend to focus on recent measurements, and in most cases the experiments have released far more detailed documentation than I could possibly work into proceedings. So what's the point?

In this set of TASI lectures, however, I attempted to focus on meaning over measurements. After 8 years of LHC running, is our view of the universe any different? If so, how? The answers to such questions are of course time dependent, and I hope that near-future discoveries at the LHC render these proceedings quickly obsolete. Nevertheless, in the hope that there is some value in this, I've adopted a similar strategy to the proceedings as in the lecture: qualitative over quantitative, meaning over measurements, and understanding over facts.

If I were forced to describe the state of high-energy physics in a single sentence, it would be "the Higgs boson is telling us there is new physics at the $\mathrm{TeV}$ scale, and flavor physics is telling us there isn't." This isn't the only thing we don't understand - dark matter, dark energy and the equality of electron and proton charges are others - but it's a tension that emerges from present research, and as such there may be hope that the resolution isn't too far down the road.

Flavor constraints come from a variety of experiments: B-factories, rare kaon decay experiments, lepton magnetic moments, and many others. These constrain a large number of models: the basic idea is that new fields introduce new amplitudes, and if these amplitudes are not exactly proportional to the Standard Model (SM) amplitudes, deviations in observables will occur. The lack of incontrovertible evidence (there are some 2-3 sigma effects) tells us that new physics that affects flavor needs to be at a scale above $10-30 \mathrm{TeV}$. This is a somewhat squishy number: because new physics effects typically are inversely proportional to the fourth power of the new scale, this factor of 3 is actually a factor of 100 in rate. However, flavor usually involves sines and/or cosines of mixing angles, and the product of several of these can easily be .01 or even lower.

On the other hand, the mass of the observed Higgs boson of $125 \mathrm{GeV}$ implies new physics at what is usually called the "TeV scale" but should probably be called the "electroweak scale" around $246 \mathrm{GeV}$, the Higgs vacuum expectation value, significantly lower than $1 \mathrm{TeV}$. Hence the tension with the equivalent number of $10-30 \mathrm{TeV}$ for flavor. The Higgs boson is telling us there is new physics at the TeV scale, and flavor physics is telling us there isn't.

The difficulty presented by a Higgs boson mass of $125 \mathrm{GeV}$ is that the Higgs mass is subject to large radiative corrections: corrections that naturally drive the Higgs mass to the highest scale in the theory, such as the GUT scale or the Planck scale. A Higgs bare mass that happened to coincidentally cancel these corrections - to something like 34 decimal places - is a wholly unsatisfying explanation. Theorists search for better explanations, such as supersymmetry, which reduce the radiative corrections by having them exactly (for an unbroken supersymmetry) cancel between SM particles and the new particles introduced in the theory. We know that supersymmetry, if realized in nature, is broken because we haven't seen any supersymmetric particles, but if we want this cancellation to be good to the electroweak scale, the lightest of the supersymmetric particles needs to have a mass comparable to this scale. Where are they? While the LHC searches are far from complete - $99 \%$ of the data is yet to come - the searches are already setting limits above the electroweak scale, at or near the $\mathrm{TeV}$ scale, and in some cases at the few $\mathrm{TeV}$ scale.

It's a bit unfair to claim the hierarchy problem - the subject of the previous paragraph - as 
something discovered by the LHC. This problem was known long before the LHC turned on: for the Higgs boson to do its job, it needs to weigh less than about $800 \mathrm{GeV}$, so you have this problem whether you have discovered the Higgs or not. Nevertheless, the discovery of the Higgs boson has promoted this from a purely theoretical problem to one that is grounded in experiment.

What else would we like to know about the Higgs boson? For starters, how many of these are there? Nature admits one (presumably) fundamental scalar. Why not more? While I'd like to say that the absence of a signal of a 2nd Higgs boson tells us something, I don't think it does.

Our measurements tell us that the Higgs boson at $125 \mathrm{GeV}$ has couplings consistent with the SM, but our ability to measure is typically at the 30more like the SM than the other. Finding that one, and seeing that it matches to within 30terribly powerful constraint on the other. Furthermore, at $125 \mathrm{GeV}$, the decay to two photons (the most powerful single search channel) is at its largest. A second Higgs will have a smaller production cross-section due to the reduced couplings, and a smaller branching fraction into the most favorable modes. This one-two punch makes finding additional Higgs bosons hard, and discovery this early in the program would require substantial optimism. Nevertheless, the LHC needs to continue looking. Its job is to count the number of Higgs bosons and so far we're still at "one".

Two paragraphs up, I wrote "presumably fundamental". While there is no evidence that the Higgs boson is composite, there is a hint that it's not. The hint might even be elevated to "clue". The top quark Yukawa is, to within our ability to measure, exactly equal to one. Is this telling us that the top quark is playing a special role here? Maybe it is. When the top quark was discovered, people were asking "why is it so heavy?" Perhaps the real question is "why is everything else so light?" And if the waters weren't muddy enough, it's worth pointing out that all of the information we have about Higgs fermion couplings comes from third-family fermions. We know nothing at all about the interactions with the first two families.

Besides the Higgs-flavor tension, the Higgs boson is telling us something else about flavor. It's telling us that there are exactly three families of quarks and leptons. There is no sequential fourth family. Ninety percent of Higgs boson production at the LHC is through gluon fusion via a virtual top quark loop. A fourth family would add two more such loops from the fourth family charge $+2 / 3$ and $-1 / 3$ quarks. Usually having a heavy fermion in a loop causes it to decouple as the mass gets large, but that is not the case here, because the Higgs Yukawa coupling grows with mass. Instead, these loops tend towards a constant at high mass. The net effect is that the amplitude triples, so the rate increases by a factor of nine. Nothing like this is observed.

I don't understand why nature has exactly three sequential families of fermions. Zero, one and infinity are numbers I understand. Three is not.

There are of course, loopholes. This is an argument relevant for chiral families. Nature could certainly have vector-like fermions. I wouldn't call this a "fourth family" since its character is totally unlike the other three, but it could be there. A possibly more interesting loophole is if there are multiple Higgs bosons, with different Higgs bosons coupling differently to different families. While that, in and of itself, wouldn't explain anything, it would be a clue about how the various pieces of the puzzle might be arranged. In the SM, the CKM matrix is a manifestation of the Higgs Yukawa couplings. Perhaps there's more to it than that.

My third topic was a brief report on the decay of $B_{s}$ mesons to dimuons. I picked this for a number of reasons. One was to provide an example of multiple LHC experiments working to- 
gether to achieve sensitivities that neither could on its own. A second was to show yet another supersymmetry search that turned out negative. Many but not all TeV-scale SUSY models will alter this branching fraction, so this is yet another example of flavor physics pointing at a scale above the electroweak scale. It was also a way to sneak in my favorite way of describing what we leaned: that an amplitude for any new process can be no larger than about half that of the Standard Model. In the SM, this is a GIM-suppressed, second-order weak process, so it's small. But it's not unmeasurably so - I showed you a measurement - so that is roughly the sensitivity of this channel.

The summary is supposed to be upbeat, with a basic message of "Go get 'em, tigers!" One might think that's not the case here. Sure, I listed some progress, starting with the Higgs boson discovery, but the central theme is one of tension. Yet this is a good thing! We make progress in science not by a parade of measurements that exactly agree with our theories. We make progress when we have disagreements and things that just don't seem to fit together. ThatâĂŹs the picture that's starting to emerge: Higgs physics (or more generally electroweak physics) is pointing us in one direction, and flavor physics is pointing us in another. Fortunately, this is happening just as we are able to make the measurements to shed light on this: most of the LHC data is yet to come, and the next generation of flavor physics experiments is coming on line now or the near future.

\section{2. $Q$ and $A$}

One question I was asked was whether I saw the logical next step to be a large circular collider in China. This is really two questions in one "should the next step be a large proton collider?" and "where should it be located?" On the first question, I believe the next step still needs some input from the LHC. There are scenarios, even today, where the next logical follow-up accelerator should be a linear electron-positron collider. In others, it might be a larger proton collider. In still others, it may be wisest to look at other things entirely: dark matter searches or tests of fundamental symmetry. The second question is not a scientific one. It is true that for many years China has sent their students outside China for education, and many of these students have remained there, to the benefit of the host countries. China obviously would like a world in which other countries send their best students to China to be educated, with some remaining there, and their investments in science and technology bear them out. Is the right area for them to focus on particle physics? That's a different and difficult question, one where I have no special insight.

Another was a request for comment on the President's budget request for high energy physics, which was $\$ 672,000,000$, which can be compared to $\$ 825,000,000$ for the previous fiscal year. In the United States, the budget originates in Congress (constitutionally in the House of Representatives), and since TASI it has been made clear that Congress has very different ideas on the appropriate level of funding. Still, there is an important point to be made. A budget of $\$ 672 \mathrm{M}$ would represent a significant decrease, and would be very difficult to implement, but it is a lot of money. It's reasonable and sensible to explain these difficulties to decision-makers, but we should not adopt a tone of entitlement. Personally, I am grateful that I live in a time and place where I can make a living studying the properties of matter on the smallest scales, and part of this gratitude is due to the field of HEP being able to "give back" to the community who supported this. I believe that is not only the right way to look at things, but also the most effective. 